Eine Replik

\title{
Wie kann Exnovation politisch gestaltet werden?
}

\author{
In der Ausgabe 01/2017 von ÖkologischesWirtschaften skizzierte \\ Dirk Arne Heyen Ansätze für eine politische Gestaltung von \\ Exnovation. Doch eine solche konsensual und langfristig \\ getroffene Entscheidung zum Ausstieg aus fossiler Energie- \\ erzeugung ist wahrscheinlich nicht ausreichend. \\ Von Martin David
}

D er Artikel „Politische Gestaltung von Exnovation" widmet sich dem vollständigen Ausstieg aus nicht nachhaltigen Technologien, „wie sie bei der Kernenergie derzeit abläuft und künftig etwa auch für Kohle, fossile Kraftstoffe oder Fluorkohlenwasserstoffe (FKW) nötig erscheint (Heyen 2017, S. 30)." Die in jedem Falle zu bejahenden Befunde, dass man „Alternativen fördern“ und für den Ausstieg „Unterstützung finden“ (ibidem, S. 31) muss und es überdies „Unterstützung für Betroffene" des Ausstiegs (ebd. S. 33) geben sollte, lassen sich gut auf die policy-termination-Literatur zurückführen, an die Heyen (2017, S. 30) Exnovationsprozesse konzeptionell knüpft (siehe insbesondere Bardach 1976; deLeon 1978; Bauer et al. 2012). Allerdings bieten zwei Punkte Grund zum Überdenken des eher rationalen Handlungsmodells, das diesem Literaturstrang zugrunde liegt.

Zum einen betrifft das die vom Artikel selbst kritisch ins Spiel gebrachte Formel konsensualer Exnovationsentscheidung („Ausstieg im Konsens?“ ibidem, S. 32). Gerade die deutsche Kohleförderpolitik war Zeit ihres Bestehens (seit den 1950er Jahren) bis zu ihrer Beendigung Gegenstand kontroverser Debatten (Meyer et al. 2010), nicht nur im Hinblick auf die parallele Förderung von Nuklearenergie in Deutschland (Curli 2017) oder KyotoZiele (Lauber/Jacobson 2015). Bis Anfang der 1980er Jahre wurden Kohlesubventionen zudem als notwendiges Übel zum Erhalt einer Brückentechnologie in einer nachhaltigkeitsorientierten Energiewende mit dem ursprünglichen Ziel ei- ner Kernenergie-Exnovation akzeptiert (Krause et al. 1980). Hier drängt sich die Frage nach der Zeitlichkeit einer Konsensualpolitik auf, denn der gesellschaftliche Diskurs des vom Artikel angebrachten Beispiels der deutschen Energiewende hat sich stets gewandelt und damit auch langfristige Voraussetzungen etwaiger konsensualer Ausstiegsszenarien. Der zickzackförmig verlaufene Politikprozess des deutschen Atomausstiegs sowie die seit den 1970er Jahren kontrovers geführte Diskussion des deutschen Atomenergieausbaus verdeutlichen das (Maubach 2014; Lauber/Jacobson 2015; Renn/ Marshall 2016; Kungl/Geels 2017).

Zum anderen muss die im Artikel vorgeschlagene langfristige Planbarkeit von Exnovationsprozessen („Zeitlich gestreckt, aber frühzeitig eingeleitet", ibidem, S. 33) mit dem empirischen Hintergrund der deutschen Energiewende eigentlich hinterfragt werden. $\mathrm{Ob}$ die Kohlekrise in den 1950er Jahren (Graichen 2003), die Ölkrisen in den 1970er Jahren (Renn/Marshall 2016), die plötzliche politische Stärke der Anti-Atombewegung der 1970er Jahre (Maubach 2014), die bestätigenden Desaster Tschernobyl und Fukushima (Lauber/Jacobsson 2015), die deutsche Wiedervereinigung oder Finanzkrisen: Eine langfristige Planung des deutschen Energiesystems wurde stets durch Unvorhersehbarkeiten infrage gestellt. Dabei ist von der abenteuerlichen Entwicklung des Innovationsinstruments Erneuerbare-Energien-Gesetz (EEG) ganz zu schweigen (siehe insbesondere Lauber/Jacobsson 2015).
Zudem ist offen, ob Großtechnologien, die historisch nicht partizipativ oder konsensual angelegt wurden, auf Grundlage einer langfristig geplanten, partizipativ und konsensual getroffenen Entscheidung abgeschafft werden können.

Der alleinige Maßstab politisch konsensualer Entscheidungsfindung zur Konfliktvermeidung innerhalb eines politischen Systems reicht wahrscheinlich nicht aus, um so komplexe und so konfliktiv verlaufende gesellschaftliche Prozesse wie Exnovationen von Großtechnologien zu erklären. Ferner sollte in Erwägung gezogen werden, dass Konflikte per se Treiber für Technologieentwicklung (siehe insbesondere Hård 1993), und somit wahrscheinlich auch für Exnovationen sind. Die eher modernistische Lesart von Exnovation als langfristig planbaren Prozess, der Erneuerungen anstößt und durch diese gleichzeitig verstärkt wird [1], würde von der Öffnung hin zur Frage profitieren, von wessen Exnovationskonsens eigentlich konkret die Rede ist und welche Mittel bestehen, Exnovationsprozesse partizipativer zu gestalten [2].

\section{Anmerkungen}

[1] So unterstreicht es die einschlägige Exnovationsliteratur bereits lange (insbesondere Kimberly 1981, Kimberly/Evanisko 1981).

[2] Der Autor dankt dem BMBF zur Förderung der Nachwuchsgruppe GORmin - Governanceoptionen für die akzeptable Gewinnung wirtschaftsstrategischer Ressourcen aus primären und sekundären Lagerstätten in Deutschland (Fördernummer: 033R148).

\section{Literatur}

Das komplette Literaturverzeichnis des Artikels ist unter 10.14512/OEW320410 abrufbar.

AUTOR + KONTAKT

Dr. Martin David ist wissenschaftlicher Mitarbeiter im Department Stadt- und Umweltsoziologie am Helmholtz-Zentrum für Umweltforschung - UFZ in Leipzig. Helmholtz-Zentrum für Umweltforschung GmbH - UFZ, Department Stadt- und Umweltsoziologie, Permoserstr. 15, 04318 Leipzig. Tel.: +49341 235-4741. E-Mail: martin.david@ufz.de 